\title{
ENTEROBACTERIAS PRODUCTORAS DE BETALACTAMASAS DE ESPECTRO EXTENDIDO EN MUESTRAS FECALES EN EL INSTITUTO NACIONAL DE SALUD DEL NIÑO, PERÚ
}

\author{
Fabiola Colquechagua Aliaga ${ }^{1, a}$, Carlos Sevilla Andrade ${ }^{1, a}$, Edgar Gonzales Escalante ${ }^{1,2, a, b}$
}

\author{
RESUMEN
}

Objetivos. Describir la frecuencia de enterobacterias productoras de betalactamasas de espectro extendido (BLEE) en muestras fecales en el Instituto Nacional de Salud del Niño, Lima, Perú. Materiales y métodos. Se analizaron muestras de heces recibidas entre julio de 2012 y marzo de 2013, se trabajó con colonias sospechosas de ser enterobacterias productoras de BLEE que se desarrollaron en el agar Karmali; se realizó la identificación bioquímica por métodos convencionales y la confirmación del fenotipo BLEE. El análisis genotípico para detectar el gen de betalactamasa de la familia CTX-M se realizó por PCR. Resultados. De 235 muestras fecales se aisló un 64,2\% de enterobacterias productoras de BLEE siendo Escherichia coli 86,1\%, Klebsiella pneumoniae 7,9\%, Salmonella sp. $2,6 \%$, Enterobacter cloacae 2,0\% y Proteus mirabilis 1,3\%. El $89,1 \%$ de las enterobacterias productoras de BLEE presentaron el gen $b / a_{C T X-M}$. Se encontró una alta resistencia al ácido nalidíxico $84,8 \%$, ciprofloxacina $74,2 \%$ y trimetoprimsulfametoxazol $81,5 \%$. La resistencia a la amikacina fue de $1,3 \%$ y todos los aislados fueron sensibles al imipenem y meropenem. Conclusiones. Se encontró una alta frecuencia de enterobacterias productoras de BLEE en muestras fecales de pacientes ambulatorios atendidos en los consultorios externos y emergencia del Instituto Nacional de Salud del Niño en Perú.

Palabras clave: beta-Lactamasa I; Heces; Enterobacterias (fuente: DeCS BIREME).

\section{EXTENDED-SPECTRUM BETA-LACTAMASE (ESBL)-PRODUCING ENTEROBACTERIACEAE IN FECAL SAMPLES AT THE NATIONAL INSTITUTE OF CHILD HEALTH, PERU}

\begin{abstract}
Objectives. To describe the frequency of extended-spectrum beta-lactamase (ESBL)-producing enterobacteriaceae in fecal samples at the National Institute of Child Health, Lima, Peru. Materials and methods. Stool samples received between July 2012 and March 2013 with colonies suspected to be ESBL-producing enterobacteriaceae that developed in Karmali agar were analyzed. Conventional methods were performed for biochemical identification and the confirmation of the ESBL phenotype. Genotypic analysis to detect the beta-lactamase gene CTX-M family was performed by PCR. Results. Of the 235 fecal samples analyzed, $64.2 \%$ of ESBL-producing enterobacteria was isolated being $86.1 \%$ Escherichia coli, $7.9 \%$ Klebsiella pneumoniae, 2.6\% Salmonella sp, 2.0\% Enterobacter cloacae, and $1.3 \%$ Proteus mirabilis. $89.1 \%$ of the ESBL-producing enterobacteria presented the CTX-M gene. We found high resistance to nalidixic acid $84.8 \%, 74.2 \%$ ciprofloxacin and trimethoprim-sulfamethoxazole $81.5 \%$. The resistance to amikacin was $1.3 \%$ and all isolates were susceptible to imipenem and meropenem. Conclusions. A high frequency of ESBL-producing enterobacteria was found in fecal samples of outpatients seen in the outpatient and emergency departments of the National Institute of Child Health of Peru.
\end{abstract}

Key words: beta-Lactamasa I; Feces; Enterobacteria (source: MeSH NLM).

\footnotetext{
Laboratorio de Epidemiología Molecular y Genética, Instituto de Medicina Tropical, Universidad Nacional Mayor de San Marcos. Lima, Perú. Instituto Nacional de Salud del Niño. Lima, Perú.

Tecnólogo médico; ${ }^{\mathrm{b}}$ magíster en Microbiología

Recibido: 30-06-14 Aprobado: 10-12-14
}

Citar como: Colquechagua Aliaga F, Sevilla Andrade C, Gonzales Escalante E. Enterobacterias productoras de Betalactamasas de espectro extendido en muestras fecales en el Instituto Nacional de Salud del Niño, Perú. Rev Peru Med Exp Salud Publica. 2015;32(1):26-32. 


\section{INTRODUCCIÓN}

La resistencia a los antibióticos constituye un problema serio de salud pública en el mundo, el cual se ha agudizado durante los últimos años, ya que a pesar de disponer nuevos antibióticos, el ritmo del desarrollo de la resistencia bacteriana en los diferentes patógenos, representa un constante desafío terapéutico ${ }^{(1)}$.

La resistencia a antibióticos pueden atribuirse a diferentes mecanismos, sin embargo, el mecanismo más frecuente e importante, desde el punto de vista terapéutico, en bacilos Gram negativos es la producción de enzimas tipo betalactamasas ${ }^{(2,3)}$. Las infecciones causadas por microorganismos productores de betalactamasas de espectro extendido (BLEE) son difíciles de manejar y la terapia empírica a menudo fracasa; además, estos organismostienden a ser resistentes aotros antimicrobianos incluyendo las fluoroquinolonas y aminoglucósidos ${ }^{(4,5)}$.

Hasta hace unos años se consideraba que los microorganismos productores de BLEE eran un problema que se daba mayoritariamente en las instituciones hospitalarias. Sin embargo, en la actualidad muchas investigaciones describen que estos microorganismos resistentes han salido del perímetro hospitalario, presentándose casos comunitarios ${ }^{(6)}$.

El tracto digestivo humano ofrece el ambiente adecuado a ser colonizado por enterobacterias productoras de BLEE. Cuando las bacterias del contenido fecal, por diversas situaciones clínicas, invaden tejidos o cavidades estériles pueden diseminarse por todo el organismo provocando septicemia, shock, infección del tracto urinario e infecciones del tracto respiratorio ${ }^{(6)}$. En Perú son pocos los estudios dirigidos a la búsqueda de resistencia a los antimicrobianos en bacterias propias del tracto intestinal ${ }^{(7)}$.

El presente estudio tiene como objetivo determinar la presencia de enterobacterias productoras de BLEE en muestras fecales con solicitud de coprocultivo en el Laboratorio de Microbiología del Instituto Nacional de Salud del Niño (INSN).

\section{MATERIALES Y MÉTODOS}

Estudio observacional, de corte transversal y descriptivo. La población de estudio fueron muestras fecales consecutivas no repetidas de pacientes con solicitud de coprocultivos provenientes de consultorio externo y emergencia, que presentaban gastroenteritis y llegaron al laboratorio de microbiología del Instituto Nacional de Salud del Niño en Lima, Perú entre julio de 2012 y enero de 2013.

\section{COPROCULTIVOS}

El aislamiento primario se realizó sembrando las muestras de heces en los siguientes medios de cultivo selectivos: agar Salmonella-Shigella (SS), agar xilosa lisina desoxicolato (XLD), agar tiosulfato citrato bilis sacarosa (TCBS), agar MacConkey, agar Karmali (medio selectivo para el aislamiento del género Campylobacter), según métodos convencionales estandarizados en el Laboratorio de Microbiología del INSN.

\section{BÚSQUEDA PRESUNTIVA DE ENTEROBACTERIAS PRODUCTORAS DE BLEE}

Las colonias que desarrollaron en el agar Karmali (medio que contiene $32 \mu \mathrm{g} / \mathrm{mL}$ de cefoperazona, $20 \mu \mathrm{g} / \mathrm{mL}$ de vancomicina y $10 \mu \mathrm{g} / \mathrm{mL}$ de anfotericina B), diferentes al género Campylobacter, se consideraron probables productoras de BLEE al ser resistentes a cefoperazona, una cefalosporina de tercera generación.

Se realizó la coloración Gram y la prueba de oxidasa de las colonias, los bacilos Gram negativos y oxidasa negativos fueron conservadas en caldo tripticasa de soya (TSB) con glicerol al $20 \%$ y almacenadas a $-20^{\circ} \mathrm{C}$ hasta su procesamiento.

\section{IDENTIFICACIÓN BACTERIANA}

Para la reactivación de los aislados conservados en el medio TSB, se sembraron por dispersión y agotamiento en el medio MacConkey, incubándolos a $35^{\circ} \mathrm{C}$ por 18 a $24 \mathrm{~h}$, luego se procedió a la identificación con las pruebas bioquímicas convencionales triple sugar iron (TSI), lisina hierro agar (LIA), sulfuro indol movilidad (SIM) y citrato de Simmon's.

\section{DETECCIÓN DE FENOTIPO BLEE}

La determinación de la producción de BLEE se realizó mediante el método de Jarlier ${ }^{(8)}$ (método de sinergia del doble disco), se utilizó una placa de agar Mueller Hinton (MH) y se inoculó con una suspensión bacteriana de $0,5 \mathrm{McF}$ arland, sobre ella se colocaron discos de susceptibilidad antimicrobiana (Oxoid): ceftazidima (30 $\mu \mathrm{g})$, cefotaxima $(30 \mu \mathrm{g})$ y cefepime $(30 \mu \mathrm{g})$ a $20 \mathrm{~mm}$ de centro a centro de un disco central de amoxicilina/ácido clavulánico $(20 / 10 \mu \mathrm{g})$, esta placa se incubó a $35^{\circ} \mathrm{C}$ por 18 - $24 \mathrm{~h}$; posteriormente, se realizó la lectura. La presencia de BLEE se manifestó por el efecto sinérgico entre el inhibidor y los discos de antimicrobianos ${ }^{(9)}$.

Adicionalmente, se realizó pruebas de susceptibilidad mediante el método de Kirby Bauer, siguiendo los 
lineamientos del CLSI 2013, para ello se utilizaron discos de ciprofloxacina (5 $\mu \mathrm{g})$, ácido nalidixico (30 $\mu \mathrm{g})$, gentamicina $(10 \mu \mathrm{g})$, amikacina $(30 \mu \mathrm{g})$, cefoxitina $(30 \mu \mathrm{g})$, sulfametoxazol/trimetoprim $(23,75 / 1,25 \mu \mathrm{g})$, cloranfenicol $(30 \mu \mathrm{g})$, imipenem $(10 \mu \mathrm{g})$ y meropenem $(10 \mu \mathrm{g})^{(10)}$.

\section{DETECCIÓN GENOTÍPICA DE LAS BETALACTAMASAS DE ESPECTRO EXTENDIDO}

Todas las muestras con fenotipo BLEE positivo, fueron confirmadas mediante la detección genotípica por PCR en el Laboratorio de Epidemiologia Molecular y Genética del Instituto de Medicina Tropical "Daniel A. Carrión" de la Facultad de Medicina de la Universidad Nacional Mayor de San Marcos. La obtención del ADN se realizó a partir de cultivos de $24 \mathrm{~h}$ de los microorganismos en estudio, se resuspendió de 3 a 4 colonias en $200 \mu \mathrm{L}$ de agua Milli-Q estéril. Se sometieron a ebullición durante 15 minutos y se centrifugó durante 2 min a una velocidad de $12000 \mathrm{rpm}$ para descartar los restos celulares. Se conservó el sobrenadante a $-20{ }^{\circ} \mathrm{C}$.

Los primers usados para la obtención del gen bla ${ }_{\text {CTX-M }}$ fueron: (5'- TTTGCGATGTGCAGTACCAGTAA-3') y (5'-CGATATCGTTGGTGGTGCCAT-3') correspondiente a 544 pares de bases.

Los reactivos empleados fueron de Invitrogen (EE. UU.). Se empleó un termociclador VERITI Applied Byosistem (EE. UU.) La amplificación se realizó en las siguientes condiciones: desnaturalización inicial a $94^{\circ} \mathrm{C}$ por $5 \mathrm{~min}$; desnaturalización a $94^{\circ} \mathrm{C}$ por $30 \mathrm{~s}$; hibridación a $58^{\circ} \mathrm{C}$ por $30 \mathrm{~s}$; Extensión a $72{ }^{\circ} \mathrm{C}$ por $30 \mathrm{~s}$ y extensión final a $72{ }^{\circ} \mathrm{C}$ durante $1 \mathrm{~min}$, en total 35 ciclos.

\section{ANÁLISIS DE DATOS}

Se realizó un análisis univariado de cada una de las muestras clasificándolas en productoras o no productoras de betalactamasas. Para la determinación de los porcentajes de resistencia y sensibilidad antimicrobiana de las distintas especies de enterobacterias en estudio, los datos obtenidos fueron introducidos en Microsoft Excel 2010 y en el programa WHONET (Word Health Organization Net Versión 5.6), programa estadístico utilizado por la Organización Panamericana de la Salud para la vigilancia de la resistencia bacteriana, que permitió analizar datos epidemiológicos del portador y las características del germen en estudio.

\section{CONSIDERACIONES ÉTICAS}

El protocolo del estudio fue aprobado por el Comité de Ética de la Facultad de Medicina de la Universidad
Nacional Mayor de San Marcos y del Comité de Ética del Instituto Nacional de Salud del Niño. Se siguió los criterios y normas éticas establecidas en la "Declaración de Helsinki", de acuerdo con los lineamientos de las buenas prácticas clínicas y de ética en investigación biomédica todos los procedimientos del presente estudio tratan de preservar la privacidad y los derechos fundamentales de los pacientes sujetos a investigación.

\section{RESULTADOS}

Se analizaron un total de 235 muestras de heces con solicitud de coprocultivos. El 58,7\% (138/235) de las muestras provenían de pacientes ambulatorios y el $41,3 \%$ (97/235) acudieron al servicio de emergencia. De ellos el 55,3\% (130/235) eran de sexo masculino. La edad promedio de la población estudiada fue de tres años, rango de 0 a 18 años.

\section{ENTEROBACTERIAS PRODUCTORAS DE BLEE AISLADAS EN MUESTRAS FECALES}

Se aislaron 151 enterobacterias productoras de BLEE, representando un 64,2\% (151/235) del total. La distribución según género y especie fueron Escherichia coli $86,1 \%$, Klebsiella pneumoniae 7,9\%, Salmonella sp. 2,6\%, Enterobacter cloacae 2,0\% y Proteus mirabilis 1,3\%.

La distribución de enterobacterias productoras de BLEE, de acuerdo al sexo y grupo etario de los pacientes fue: $68,7 \%$ (103/150) lactantes; 9,3\% (14/150) prescolares; $14,7 \%(22 / 150)$ escolares, y 7,3\% (11/150) adolescente. La frecuencia de enterobacterias productoras de BLEE en el sexo masculino se presenta ligeramente superior al sexo femenino (Figura 1). Solo se procesaron dos muestras de heces pertenecientes a neonatos donde no se aisló enterobacterias productoras de BLEE.

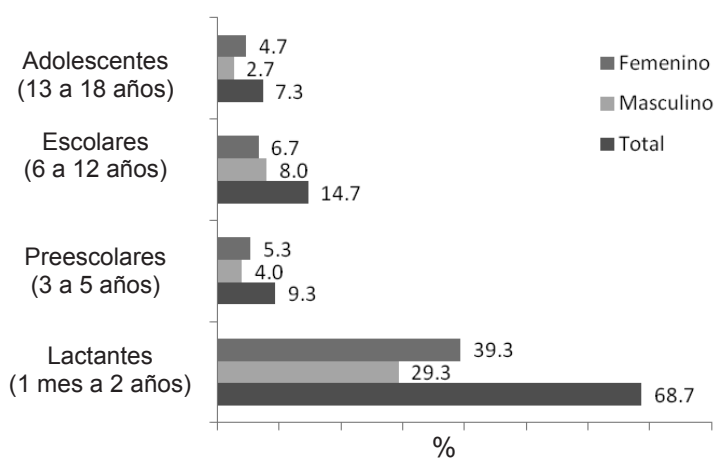

Figura 1. Distribución de enterobacterias productoras de BLEE de acuerdo al género y grupo etario de los pacientes ambulatorios. Julio 2012 a enero $2013(n=235)$ 


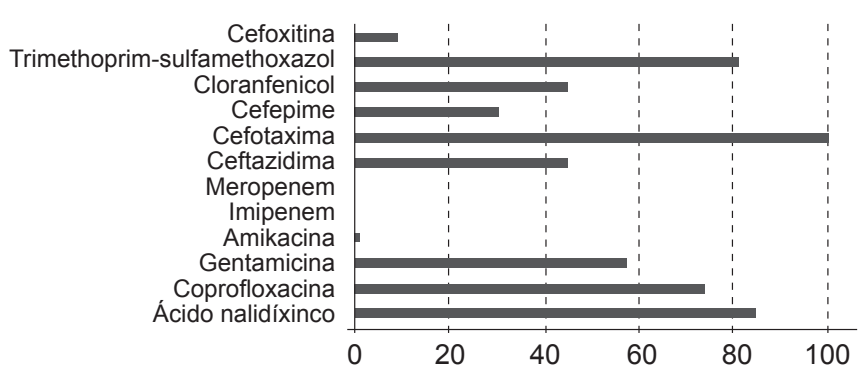

Figura 2. Resistencia de las enterobacterias productoras de BLEE aislados en muestras fecales de pacientes ambulatorios del Instituto Nacional de Salud del Niño. Julio 2012 a enero 2013

\section{ANÁLISIS DE LA SUSCEPTIBILIDAD ANTIMICROBIANA}

La susceptibilidad al ácido nalidixico, ciprofloxacina, gentamicina, cloranfenicol, trimetoprimsulfametoxazol fue variable. En el grupo de las quinolonas y fluoroquinolonas se evaluaron el ácido nalidíxico y la ciprofloxacina con una resistencia de 84,8 y $74,2 \%$ respectivamente. En el grupo de los aminoglucósidos se encontró una resistencia del $57,6 \%$ para la gentamicina y $1,3 \%$ a la amikacina. La resistencia al trimetoprim-sulfametoxazol fue alta en todos los aislamientos con el $81,5 \%$. La resistencia al cloranfenicol fue $45,0 \%$ y la sensibilidad $47,0 \%$. Todos los aislados fueron sensibles a los carbapenémicos. Se observa una corresistencia a 5 clases de antibióticos (quinolonas, fluoroquinolonas, aminoglucósidos, trimetoprim-sulfametoxazol y cloranfenicol) en el $88,7 \%$ de los aislamientos (Figura 2 ).

\section{ANÁLISIS GENOTÍPICO MEDIANTE PCR}

Según el análisis molecular $123(89,1 \%)$ de los 138 aislamientos de las enterobacterias productoras de BLEE presentaron el gen $b / a_{\text {СTX-M. }}$. Las enterobacterias productoras de BLEE aisladas fueron: Escherichia coli 86,2\% (119/138); Klebsiella pneumoniae 7,9\% (11/138); Salmonella sp. 2,9\% (4/138); Enterobacter cloacae $1,4 \%(2 / 138)$ y Proteus mirabilis $1,4 \%(2 / 138)$.

\section{DISCUSIÓN}

Se evidencia una alta frecuencia de enterobacterias productoras de BLEE en muestras fecales, la presencia de aislamientos resistentes de enterobacterias comensales en la flora intestinal indicaría que los niños pueden servir como un reservorio de genes de resistencia que se encuentran localizados en plásmidos altamente transmisibles; esta condición se asocia con casi el doble de la mortalidad en comparación con las no productoras de BLEE (11). La colonización intestinal por enterobacterias resistentes es un factor clave en la epidemiologia de las enterobacterias productoras de $\operatorname{BLEE}(12,13)$.

Este estudio indicó una frecuencia de $64,2 \%$ de enterobacterias productoras de BLEE, comparable a un estudio similar realizado en El Cairo en 2011, con $63,3 \%$ de bacterias productoras de BLEE, a partir de muestras de heces de 632, donde para la selección de enterobacterias sospechosas de BLEE se utilizó agar MacConkey suplementado con cefotaxima $y$ confirmados por el método del CLSI, mientras que en este estudio se utilizó el agar Karmali que contiene cefoperazona y se confirmó con el método sinergia del doble disco ${ }^{(14)}$. Los resultados encontrados difieren a los trabajos realizados por otros autores: Husickova et al. en República Checa, reportaron el 3,2\% de portadores fecales de enterobacterias productoras de BLEE en sujetos de la comunidad (15). Adriatahina et al. en una unidad pediátrica de Madagascar, realizaron hisopados rectales el primer y el último día de hospitalización, se encontró un $21,2 \%$ de productoras de BLEE en las del primer día y al momento del alta se encontró el $57,1 \%$ ${ }^{(16)}$. Estudios de Woerther et al. en niños hospitalizados en Nigeria, obtuvo una frecuencia de portadores de BLEE del $31,0 \%$ al ingreso, todos recibieron antibióticos durante su hospitalización, al momento del alta la tasa de adquisición fue del 94,0\%, donde el gen bla CTX-M-15 $_{\text {. }}$ fue encontrado en más del $90,0 \%$ de los portadores ${ }^{(17)}$.

Se observa con el avance del tiempo un incremento de la resistencia según lo reportado por: Pallechi et al. en un estudio realizado en la selva de Perú y el chaco Boliviano donde detectaron enterobacterias productoras de BLEE en $0,1 \%(4 / 3208)$ de las muestras fecales de niños sanos con presencia del gen $b l a_{C T X-M}$. El año 2005 en la misma población de niños sanos de PerúBolivia reveló el incremento de portadores fecales, en comparación al $2002(0,1 \%$ el año 2002 y 1,7\% en el 2005) ${ }^{(18,19)}$. Así como, Valverde et al. en un estudio en España informaron que las tasas de portadores fecales de enterobacterias productoras de BLEE se incrementaron de un $0,3 \%$ en 1991 a 5,5\% en el 2003 en pacientes ambulatorios y un $3,7 \%$ en voluntarios sanos el año $2003^{(20)}$. Además, se sabe por Muzaheed, et al, en un estudio realizado en portadores fecales de la India que todos los aislados resultaron ser resistentes al menos a una de las cefalosporinas de tercera generación, revelándose que Klebsiella pneumoniae y Escherichia coli son productores de betalactamasas tipo CTX-M-15 (2). 
Los factores de riego han sido estudiados por, Abdurrahman et al. donde incluyeron tratamiento previo con cefalosporinas, penicilinas y fluoroquinolonas (21). En nuestro estudio, la frecuencia de resistencia a las fluoroquinolonas es alta. Aunque no se pudo obtener la documentación que confirma la reciente exposición a los antibióticos entre los pacientes, es probable que la venta libre de antibióticos en países en desarrollo cree un fondo general de organismos resistentes en la población. Las formulaciones orales de amoxicilina clavulánico y fluoroquinolonas (ciprofloxacina y levofloxacina) son algunos de los antibióticos que con frecuencia son obtenidos y utilizados sin receta médica. Abdurrahman et al. en Turquía evaluaron la prevalencia y los factores de riesgo en portadores fecales de enterobacterias productoras de BLEE en niños hospitalizados y ambulatorios mostraron que el $7,2 \%$ de los niños ambulatorios eran portadores fecales de bacterias productoras de BLEE, dentro de los factores de riesgo se mencionan el uso indiscriminado de cefalosporinas de segunda, tercera generación y la ingesta de alimentos contaminados con bacterias resistentes ${ }^{(22)}$.

El resultado de nuestro estudio fue similar a un estudio en Egipto ${ }^{(14)}$, pero superior a la mayoría de otros informes anteriores ya mencionados. La alta resistencia mostrada puede deberse a que los antibióticos betalactámicos son ampliamente utilizados en la terapia antibacteriana a pacientes internados y de consulta externa lo que selecciona bacterias resistentes, además de la automedicación de los individuos de la comunidad.

Para facilitar la búsqueda presuntiva de enterobacterias productoras de BLEE en otros estudios utilizaron agar MacConkey suplementado con ceftazidima y cefotaxima 19), ambas cefalosporinas de tercera generación, agar Chrom ID con cefotaxima, agar Drigalski con ceftriaxona, agar EMB suplementado con cefotaxima ${ }^{(16)}$ y en el presente estudio se utilizó el medio selectivo Karmali, que contiene $32 \mu \mathrm{g} / \mathrm{mL}$ de cefoperazona, donde el desarrollo de colonias diferentes al género Campylobacter se consideraban probables productoras de BLEE al ser resistentes a cefoperazona (cefalosporina de tercera generación) lo cual fue confirmada fenotípicamente, este último medio a diferencia de los otros se puede utilizar en la rutina de coprocultivos por que permite el aislamiento del género Campylobacter.

La multidrogorresistencia (MDR) es muy común en las bacterias productoras de BLEE; en el presente estudio se encontró que el $88,7 \%$ de los productores de BLEE aislados se clasificaron como MDR de acuerdo con la definición propuesta por Magiorakos et al. (23). En las enterobacterias productoras de BLEE aisladas se observa alta resistencia acompañante a las quinolonas, fluoroquinolonas, sulfametoxazol/trimetoprim y una resistencia menor a gentamicina, cloranfenicol, siendo sensibles la amikacina y los carbapenémicos. Esto es alarmante, porque no se dispone de muchas alternativas terapéuticas, en caso de infección por alguno de estos microorganismos se puede administrar como tratamiento amikacina y en infecciones complicadas los carbapenémicos.

Cabe destacar que en seis enterobacterias productoras de BLEE, al interpretar el antibiograma se observó la disminución del halo del disco de cefoxitina $(\leq 18 \mathrm{~mm}$ ) lo que sugirió la presencia de una betalactamasa tipo AmpC, lo que se comprobó mediante el método de Hodge y utilizando el disco de ácido fenil borónico (AFB), inhibidor de las betalactamasas tipo AmpC.

En el presente estudio la frecuencia de enterobacterias productoras de BLEE fue alta en el grupo de los lactantes, este dato indicaría que la colonización con bacterias productoras de BLEE ocurre a temprana edad, puede ser por el uso cada vez mayor de los antibióticos en la comunidad o previas hospitalizaciones, lo que da lugar a una presión selectiva a favor de las bacterias que han adquirido resistencia.

Es importante mencionar que en los últimos años se ha descrito el incremento de enterobacterias productoras de BLEE de la familia CTX-M (19), en este estudio se encontró que la gran mayoría de los aislados productores de BLEE presentaban en gen bla ${ }_{C T X-M}$ el grupo de gen dominante en los estudios de portadores fecales de República Checa ${ }^{(15)}$, España ${ }^{(20)}$, India ${ }^{(2)}$, Madagascar ${ }^{(16)}$, Nigeria ${ }^{(17)}$, Francia ${ }^{(24)}$, Guinea-Bissau ${ }^{(25)}$ y Japón ${ }^{(26)}$ poniendo de relieve la importancia de este gen en la difusión global de BLEE. La BLEE tipo CTX-M tiene principalmente actividad sobre la cefotaxima y no sobre la ceftazidima; aunque en los últimos años la emergencia de variantes de CTX-M (CTX-M-15, CTX-M-16, CTX-M-27 y CTX-M-19) están mejorando su actividad frente a ceftazidima.

Según las recomendaciones del CLSI desde el año 2010 no se debería buscar enterobacterias productoras de BLEE, solo se debe informar la interpretación según los puntos de corte, pero con los resultados obtenidos en el presente estudio se demuestra que esto representaría una falla en el tratamiento, debido a que las bacterias productoras de BLEE pueden presentar halos de inhibición sensibles para ceftazidima según el punto de corte del CLSI 2013, pero ser productoras de BLEE. Por lo tanto, se debe continuar con la búsqueda, reporte e 
informe de BLEE en las pruebas de rutina, si es BLEE positivo informar resistentes a todas las penicilinas, cefalosporinas y monobactámicos, independientemente de las zonas de inhibición obtenidas, si es BLEE negativo informar según el punto de corte CLSI 2013.

Una de las limitaciones del estudio fue el prescindir de datos clínicos por la cual se solicitaron los coprocultivos y uso previo de antibióticos u hospitalizaciones. Lo que podría explicar la alta frecuencia de enterobacterias productoras de BLEE.

En conclusión, las infecciones causadas por bacterias productoras de BLEE representan un reto para el equipo de salud por la limitada disponibilidad de opciones de tratamiento, esto obliga a la prevención de estas infecciones mediante la restricción del uso de agentes antimicrobianos, junto con la aplicación de medidas inmediatas de control de infecciones. Para controlar o reducir la elevada tasa de portadores de estos microorganismos se deben tomar medidas efectivas como evitar la venta de antibióticos sin receta médica y concientizar en la población los peligros de tomar antibióticos sin consulta médica.

Agradecimientos: al personal del Servicio de Microbiología del Instituto Nacional de Salud del Niño y del Laboratorio de Epidemiología Molecular y Genética del Instituto de Medicina Tropical de la Universidad Nacional Mayor de San Marcos, por el apoyo brindado para la realización de esta investigación.

Contribuciones de autoría: FCA, RSA y EGE han participado en la idea de la investigación, concepción del artículo, la recolección de datos, material de estudio y redacción del artículo. Todos los autores aprobaron la versión final del manuscrito.

Fuentes de financiamiento: Fondo de Promoción de Trabajos de Tesis financiado por la Facultad de Medicina de la Universidad Nacional Mayor de San Marcos.

Conflictos de interés: los autores declaran no tener conflictos de interés.

\section{REFERENCIAS BIBLIOGRÁFICAS}

1. Organización Mundial de la Salud. Movilizar la voluntad política para contener la resistencia a los antimicrobianos. Bulletin of the World Health Organization. 2011;89:168-9.

2. Lahey Clinic. B-Lactamase Classification and Amino Acid Sequences for TEM, SHV and OXA Extended-Spectrum and Inhibitor Resistant Enzymes. Burlington, MA: Lahey Clinic Foundation; 2013.

3. Bush K, Jacoby GA.Updated functional classification of beta-lactamases. Antimicrob Agents Chemother. 2010 Mar;54(3):969-76. doi: 10.1128/ AAC.01009-09.

4. Muzaheed, Doi Y, Adams-Haduch JM, Shivannavar CT, Paterson DL, Gaddad SM. Faecal carriage of CTX-M-15 producing Klebsiella pneumoniae in patients with acute gastroenteritis. Indian J Med Res. 2009 May;129(5):599-602.

5. Perozo M, Armindo J, Castellano MJ. Detección de Betalactamasas de Espectro Extendido en cepas de la familia Enterobacteriaceae. Kasmera. 2009;37(1):25-37.

6. García-Hernández AM, GarcíaVázquez E, Hernández-Torres A, Ruiz J, Yagüe G, Herrero JA. Bacteriemias por Escherichia coli productor de B-lactamasas de espectro extendido (BLEE): significación clínica y perspectivas actuales. Rev Esp Quimioter. 2011 Jun;24(2):57-66.

7. Mosquito S, Ruiz J, Bauer JL, Ochoa TJ. Mecanismos moleculares de resistencia antibiótica en Escherichia coli asociadas a diarrea. Rev Perú Med Exp Salud Publica. 2011;28(4):648-56.

8. Jarlier V, Nicolas MH, Fournier G, Philippon A. Extended broadspectrum beta-lactamases conferring transferable resistance to newer betalactam agents in Enterobacteriaceae: hospital prevalence and susceptibility patterns. Rev Infect Dis. 1988 JulAug;10(4):867-78.

9. Lezameta L, Gonzáles-Escalante E, Tamariz JH.. Comparación de cuatro métodos fenotípicos para la detección de beta-lactamasas de espectro extendido. Rev Perú Med Exp Salud Pública. 2010 Sep;27(3):345-51.

10. Clinical and Laboratory Standard Institute. Performance Standards for Antimicrobial Susceptibility Testing. Twenty-third Informational Supplement M100-S23. Wayne, Pennsylvania: Clinical and Laboratory Standard Institute; 2013.
11. Schwaber MJ, Carmeli Y. Mortality and delay in effective therapy associated with extended-spectrum beta-lactamase production in Enterobacteriaceae bacteraemia: a systematic review and meta-analysis. J Antimicrob Chemother. 2007 Nov;60(5):913-20.

12. Rodríguez D, Pérez M, Sarmiento F, Díaz J, Ruiz A. Colonización del tracto digestivo en niños después de infección por gérmenes productores de betalactamasas de espectro extendido y tratamiento con carbapenems, estudio prospectivo. Infect. 2011;15(3):155-9.

13. Luvsansharav UO, Hirai I, Nakata A, Imura K, Yamauchi K, Niki M, et al. Prevalence of and risk factors associated with faecal carriage of CTX-M $\quad \beta$-lactamase-producing Enterobacteriaceae in rural Thai communities. J Antimicrob Chemother. 2012 Jul;67(7):1769-74. doi: $10.1093 / \mathrm{jac} / \mathrm{dks} 118$.

14. Abdul Rahman EM, El-Sherif RH. High rates of intestinal colonization with beta-lactamase-producing Enterobacteriaceae spread spectrum in healthy individuals. J Invest Med. 2011;59:1284-6.

15. Husickova V, Cekanova L, Chroma M, Htoutou-Sedlakova M, Hricova 
K, Kolar M. Carriage of ESBL-and AmpC-positive Enterobacteriaceae in the gastrointestinal tract of community subjects and hospitalized patients in the Czech Republic. Biomed Pap Med Fac Univ Palacky Olomouc Czech Repub. 2012 Dec;156(4):348-53. doi: 10.5507/bp.2012.039.

16. Andriatahina T, Randrianirina F, Hariniana ER, Talarmin A, Raobijaona $\mathrm{H}$, Buisson $\mathrm{Y}$, et al. High prevalence of fecal carriage of extended-spectrum B-lactamase-producing Escherichia coli and Klebsiella pneumoniae in a pediatric unit in Madagascar. BMC Infect Dis. 2010 Jul 12;10:204. doi: 10.1186/1471-2334-10-204.

17. Woerther PL, Angebault C, Jacquier H, Hugede HC, Janssens AC, Sayadi S, et al. Massive increase, spread, and exchange of extended B-lactamase-encoding genes among intestinal Enterobacteriaceae in hospitalized children with severe acute malnutrition in Niger. Clin Infect Dis. 2011 Oct;53(7):677-85. doi: 10.1093/ $\mathrm{cid} / \mathrm{cir} 522$.

18. Pallecchi L, Malossi M, Mantella A, Gotuzzo E, Trigoso C, Bartolini A, et al. Detection of CTX-M-Type B-lactamase genes in fecal Escherichia coli Isolates from Healthy Children in Bolivia and Peru. Antimicrobial Agents Chemother. 2004 Dec;48(12):4556-61.

19. Pallecchi L, Bartolini A, Fiorelli C, Mantella A, Di Maggio T, Gamboa $\mathrm{H}$, et al. Rapid Dissemination and
Diversity of CTX-M ExtendedSpectrum B-Lactamase Genes in Commensal Escherichia coli Isolates from Healthy Children from LowResource Settings in Latin America. Antimicrob Agents Chemother. 2007 Aug;51(8):2720-5.

20. Valverde A, Coque TM, SánchezMoreno MP, Rollán A, Baquero F, Cantón R. Dramatic Increase in Prevalence of Fecal Carriage of Extended-Spectrum B-LactamaseProducing Enterobacteriaceae during Nonoutbreak Situations in Spain.J Clin Microbiol. 2004 Oct;42(10):4769-75.

21. Abdurrahman K, Angamuthu K, Katapadi A. Fecal carriage of Extended-Spectrum B-lactamaseProducing Escherichia coli and Klebsiella pneumoniae in Patients and Asintomatic Healthy Individuals. J Pediatr Inf. 2007; 5:54-8.

22. Abdurrahman K, Ener C, Zeynel A, Gul D, Neslihan T, Askin D, et al. Prevalence and Risk Factors of Fecal Carriage of Extended-Spectrum [Beta]-Lactamase (Esbl)-Producing Enterobacteriaceae in Hospitalized and Ambulatory Children. J Pediatr Inf. 2011;5(2):54-8.

23. Magiorakos AP, Srinivasan A, Carey RB, Carmeli Y, Falagas ME, Giske $\mathrm{CG}$, et al. Multidrug-resistant, extensively drug-resistant and pandrugresistant bacteria: an international expert proposal for interim standard definitions for acquired resistance. Clin Microbiol Infect. 2012 Mar;18(3):26881. doi: $10.1111 /$ j.14690691.2011.03570.x.

24. Birgy A, Cohen R, Levy C, Bidet P, Comoux M, Thollot F, et al. Community fecal carriage of extendedspectrum beta-lactamase-producing Enterobacteriaceae in French children. BMC Infect Dis. 2012 Nov 21;12:315. doi: 10.1186/1471-2334-12-315.

25. Isendahl J, Turlej-Rogacka A, Manjuba C, Rodrigues A, Giske CG, Nauclér P. Fecal Carriage of ESBL-Producing E. coli and K. pneumoniae in Children in Guinea-Bissau: A Hospital-Based Cross-Sectional Study. Plos One. 2012;7(12):e51981. doi: 10.1371/ journal.pone.0051981.

26. Minami K, Shoji Y, Kasai M, Ogiso Y, Nakamura T, Kawakami Y, et.al. Proportion of rectal carriage of extended-spectrum $\quad \beta$-lactamaseproducing Enterobacteriaceae in the inpatients of a pediatric tertiary care hospital in Japan. Jpn J Infect Dis. 2012;65(6):548-50.

Correspondencia: Edgar Gonzales

Escalante

Dirección: Av. Brasil 600, Lima 05, Perú.

Teléfono: (511) 330-0066 anexo 3201

Correoelectrónico:egones_5@hotmail.com

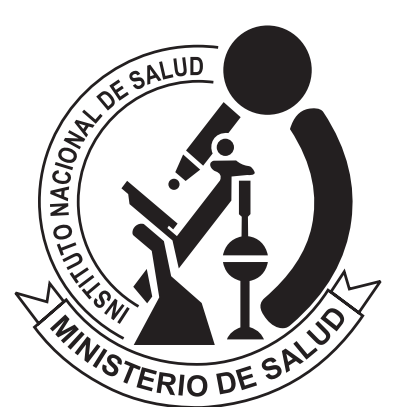

Investigar para proteger la salud
REVISTA PERUANA DE MEDICINA

EXPERIMENTAL Y SALUD PÚBLICA

CUMPLIENDO SUS METAS Y

PROYECTÁNDOSE AL FUTURO

Visite los contenidos de la revista en:
WWW.ins.gob.pe/rpmesp 